\title{
Exact Solution of Modified Veselov-Novikov Equation and Some Applications in the Game Theory
}

\author{
Damir Kurmanbayev $\mathbb{i D}^{1,2}$ \\ ${ }^{1}$ Department of Fundamental Mathematics, Al-Farabi Kazakh National University, 71 Al-Farabiave., \\ 050040 Almaty, Kazakhstan \\ ${ }^{2}$ Department of Mathematics and Natural Science, SuleymanDemirel University, 1/1 Abylai Khan str., Kaskelen, Kazakhstan \\ Correspondence should be addressed to Damir Kurmanbayev; kurmanbaev.damir@gmail.com
}

Received 21 August 2019; Revised 2 November 2019; Accepted 4 November 2019; Published 12 February 2020

Academic Editor: Dan Huang

Copyright (C) 2020 Damir Kurmanbayev. This is an open access article distributed under the Creative Commons Attribution License, which permits unrestricted use, distribution, and reproduction in any medium, provided the original work is properly cited.

A method of finding exact solutions of the modified Veselov-Novikov ( $\mathrm{mVN}$ ) equation is constructed by Moutard transformations, and a geometric interpretation of these transformations is obtained. An exact solution of the $\mathrm{mVN}$ equation is found on the example of a higher order Enneper surface, and given transformations are applied in the game theory via Kazakh proverbs in terms of trees.

\section{Introduction}

Consider the following problem with the initial data (this is the minimal surface):

$$
\begin{aligned}
\widetilde{\psi}_{t} & =A \widetilde{\psi}, \\
\left.\widetilde{\psi}\right|_{t=0} & =\psi_{0},
\end{aligned}
$$

where $A=\left(\partial^{3} / \partial z^{3}\right)$.

The following theorems are principal results of this paper.

Theorem 1. If $\psi_{0}$ is holomorphic function, then the general solution of (1)-(2) is given by the following formula:

$$
\widetilde{\psi}=\psi_{0}+\sum_{i=1}^{\infty} \int_{0}^{t} A p_{i-1} d \tau_{i-1}
$$

where $A p_{i-1}=p_{i}, p_{0}=\psi_{0}, i=1,2, \ldots$

This initial-value problem (1)-(2) is solved by the method of successive approximations [1].

Theorem 2. Let $a, b$, and $c$ be constants such that $a \gg 1, b \gg 1$, and $c \gg 1$ and

$$
|\alpha|^{2}+|\beta|^{2}=a^{2}+b^{2}+c^{2} \Longleftrightarrow x=y=0 .
$$

The Gaussian curvatures at an isolated point are not defined, but in other points, $K<0$ and $|a|+|b|+|c| \neq 0$ for all surfaces $\Sigma_{a, b, c}$ in which potentials $\widetilde{U}$ are regular.

Moreover, the inverted Enneper surface of higher order $\widetilde{S}_{a, b, c}(n \geq 3)$ is smoothly deformed into the saddle surface with surface motions $\Sigma_{a, b, c}$ by a straight line given through parameters $a, b$, and $c$.

Soliton deformation [2] of inverted Enneper surfaces will be found by parallel translation (depending on parameters $a, b$, and $c$ ) of these surfaces in the proof of Theorem 2 as the main result. Physical interpretation of exact solutions of the modified Veselov-Novikov equation (given below) corresponding to inverted Enneper surfaces analogically could be represented by solitons (depending on different parameters) for mixed coupled nonlinear Schrödinger equations [3]. Futhermore, soliton solutions were found by the generalized Darboux transformation [4].

The strategy of this work is to find the solution of the following modified Veselov-Novikov $(\mathrm{mVN})$ equation [5]:

$$
\widetilde{U}_{t}=\left(\widetilde{U}_{z z z}+3 \widetilde{U}_{z} \widetilde{V}+\frac{3}{2} \widetilde{U} \widetilde{V}_{z}\right)+\left(\widetilde{U}_{\overline{z z z}}+3 \widetilde{U}_{\bar{z}} \overline{\bar{V}}+\frac{3}{2} \widetilde{U} \widetilde{V}_{\bar{z}}\right)
$$


where

$$
\widetilde{V}_{\bar{z}}=\left(\widetilde{U}^{2}\right)_{z}
$$

where $z=x+i y$ and $\widetilde{U}(z, \bar{z}, t)$ is a real-valued function by Theorem 1 and Moutard transformations $[2,6]$ to illustrate its applications in game theory.

Taimanov [7] found blowing-up solutions of $\mathrm{mVN}$ equations by Moutard transformations on the example of a first-order Enneper surface. Theorem 1 generates all minimal surfaces (higher order Enneper surface, catenoid, and helicoid) and leads to the regular solutions of $\mathrm{mVN}$ equations formulated in Theorem 2 by given transformations.

Theorems 1 and 2 are based on the geometric interpretations of the following Moutard transformations $[7,8]$.

Theorem 3. Let $\mathscr{D}=\left(\begin{array}{cc}0 & (\partial / \partial z) \\ -(\partial / \partial \bar{z}) & 0\end{array}\right)+\left(\begin{array}{cc}U & 0 \\ 0 & U\end{array}\right)$ be the Dirac operator with potentials $U(z, \bar{z}, t)$ (that are solutions of $m V N$ equations) and $\Psi_{0}(z, \bar{z}, t)=\left(\begin{array}{cc}\psi_{1} & -\bar{\psi}_{2} \\ \psi_{2} & \bar{\psi}_{1}\end{array}\right)$ satisfy the system of equations (when $U(z, \bar{z}, t)=0$ )

$$
\begin{aligned}
& \frac{\partial \psi_{1}}{\partial \bar{z}}=\frac{\partial \bar{\psi}_{2}}{\partial \bar{z}}=0, \\
& \frac{\partial \psi_{1}}{\partial t}=\frac{\partial^{3} \psi_{1}}{\partial z^{3}}, \\
& \frac{\partial \psi_{2}}{\partial t}=\frac{\partial^{3} \psi_{2}}{\partial \bar{z}^{3}} .
\end{aligned}
$$

Then,

(1) Matrices $K\left(\Psi_{0}\right)$ and $M\left(\Psi_{0}\right)$ have the following form:

$$
\begin{aligned}
& K\left(\Psi_{0}\right)=\Psi_{0} \widetilde{S}^{-1}\left(\Psi_{0}^{T}, \Psi_{0}\right) \Gamma \Psi_{0}^{T} \Gamma^{-1}, \\
& M\left(\Psi_{0}\right)=i \Gamma\left(\Psi_{0 z}-\Psi_{0 \bar{z}}\right) \Psi_{0}^{-1} \Gamma^{-1},
\end{aligned}
$$

where $\widetilde{S}^{-1}$ is the inverse of the matrix $\widetilde{S}$ and $\Gamma=\left(\begin{array}{cc}0 & 1 \\ -1 & 0\end{array}\right)$.

(2) For every $\Psi$ solution of the system (7), $\widetilde{\Psi}$ functions are also solutions for this system and have the form $\widetilde{\Psi}=$ $\Psi-\Psi_{0} \cdot \widetilde{S}^{-1}\left(\Psi_{0}^{T}, \Psi_{0}\right) \cdot \widetilde{S}\left(\Psi_{0}^{T}, \Psi\right)$ and the Dirac operator $\widetilde{D}$ with the potential $\widetilde{U}=i k_{12}$, and $\widetilde{U}$ with the potential $\tilde{V}=\bar{k}_{11}^{2}-2\left(m_{21} k_{21}+\bar{m}_{11} \bar{k}_{11}\right)$ satisfies the following system of equations:

$$
\begin{aligned}
& \widetilde{\mathscr{D}} \widetilde{\Psi}=0, \\
& \frac{\partial \widetilde{\Psi}}{\partial t}=\tilde{\mathscr{A}} \widetilde{\Psi},
\end{aligned}
$$

where

$$
\begin{aligned}
\tilde{\mathscr{A}}= & \frac{\partial^{3}}{\partial z^{3}}+\frac{\partial^{3}}{\partial \bar{z}^{3}}+3\left(\begin{array}{cc}
\widetilde{V} & 0 \\
\widetilde{U}_{z} & 0
\end{array}\right) \frac{\partial^{3}}{\partial z^{3}}+3\left(\begin{array}{cc}
0 & -\widetilde{U}_{\bar{z}} \\
0 & \overline{\widetilde{V}}
\end{array}\right) \frac{\partial^{3}}{\partial z^{3}} \\
& +\frac{3}{2}\left(\begin{array}{cc}
\widetilde{V}_{z} & 2 \widetilde{U} \bar{V} \\
-2 \widetilde{U} \widetilde{V} & \overline{\widetilde{V}}_{\bar{z}}
\end{array}\right) .
\end{aligned}
$$

The previous matrix is chosen so that the system (9) is equivalent to Manakov's $L, A, B$ triple [5,9], which is an operator representation of $m V N$ equations.

(3) The real-valued function $\widetilde{U}$ and the function $\widetilde{V}$ satisfy the $m V N$ equations.

\section{Preliminaries}

Every pair of holomorphic functions $\psi_{1}, \bar{\psi}_{2}: \mathscr{U} \longrightarrow \mathbb{C}$ corresponds to a minimal surface $\Sigma: \mathscr{U} \longrightarrow \mathbb{R}^{3}$.

$\Sigma=\left(u^{1}, u^{2}, u^{3}\right)$ is given by the following Weierstrass representation $[7,10]$ :

$$
\begin{array}{r}
u^{1}(z, \bar{z}) \& 9 ;=\frac{i}{2} \int_{(0,0)}^{(z, \bar{z})}\left(\psi_{1}^{2}+\bar{\psi}_{2}^{2}\right) \mathrm{d} z-\left(\bar{\psi}_{1}^{2}+\psi_{2}^{2}\right) \mathrm{d} \bar{z}+u_{0}^{1}, \\
u^{2}(z, \bar{z}) \& 9 ;=\frac{1}{2} \int_{(0,0)}^{(z, \bar{z})}\left(\bar{\psi}_{2}^{2}-\psi_{1}^{2}\right) \mathrm{d} z+\left(\psi_{2}^{2}-\bar{\psi}_{1}^{2}\right) \mathrm{d} \bar{z}+u_{0}^{2}, \\
u^{3}(z, \bar{z}) \& 9 ;=\int_{(0,0)}^{(z, \bar{z})} \psi_{1} \bar{\psi}_{2} \mathrm{~d} z+\bar{\psi}_{1} \psi_{2} \mathrm{~d} \bar{z}+u_{0}^{3},
\end{array}
$$

and the initial point on the surface has some constant $u_{0}^{1}=-a, u_{0}^{2}=b$, and $u_{0}^{3}=c$.

We represent the surface in the following form [7]:

$$
\begin{aligned}
\tilde{S}= & \Gamma \Omega=\Gamma\left[\int_{0}^{z} \omega+\left(\begin{array}{cc}
a+b i & c i \\
c i & a-b i
\end{array}\right)\right] \\
= & \left(\begin{array}{cc}
i u^{3} & -u^{1}-i u^{2} \\
u^{1}-i u^{2} & -i u^{3}
\end{array}\right)-i \int_{0}^{t}\left(\begin{array}{cc}
l & \bar{k} \\
k & -l
\end{array}\right) \mathrm{d} \tau \\
& +\left(\begin{array}{cc}
c i & a-b i \\
-a-b i & -c i
\end{array}\right) \\
= & \left(\begin{array}{cc}
\alpha & -\bar{\beta} \\
\beta & \bar{\alpha}
\end{array}\right),
\end{aligned}
$$


where

$$
\begin{aligned}
& \alpha=i \int_{0}^{z} \psi_{1} \bar{\psi}_{2} \mathrm{~d} z+\bar{\psi}_{1} \psi_{2} \mathrm{~d} \bar{z}-i \int_{0}^{t} l \mathrm{~d} \tau+2 i c, \\
& \beta=i \int_{0}^{z} \psi_{1}^{2} \mathrm{~d} z-i \int_{0}^{t} k \mathrm{~d} \tau-2(a+b i), \\
& k=\psi_{1, z}^{2}-\psi_{2, \bar{z}}^{2}-2\left(\psi_{1} \psi_{1, z z}-\psi_{2} \psi_{2, \overline{z z}}\right), \\
& l=\psi_{1, z} \bar{\psi}_{2, z}+\bar{\psi}_{1, \bar{z}} \psi_{2, \bar{z}}-\psi_{1, z z} \bar{\psi}_{2}-\psi_{1} \bar{\psi}_{2, z z}-\bar{\psi}_{1, \overline{z z}} \psi_{2}-\bar{\psi}_{1} \psi_{2, \overline{z z}}
\end{aligned}
$$

\section{Solution of the $m V N$ Equation}

Let $n \geq 3$. Then, the matrix representations $\widetilde{S}_{t}$ for all inverted minimal surfaces of Enneper $\left(\Sigma_{t}\right)$ have to be presented in the following holomorphic functions:

$$
\begin{aligned}
\begin{aligned}
\psi_{1}= & z^{n}+n(n-1)(n-2) z^{n-3} t+n(n-1) \cdots(n-5) z^{n-6} \frac{t^{2}}{2 !} \\
& +n(n-1) \cdots(n-8) z^{n-9} \frac{t^{3}}{3 !}+\cdots,
\end{aligned} \\
\psi_{2}=1,
\end{aligned}
$$

with potential $\tilde{U}(z, \bar{z}, t)$ having the following representation:

$$
\widetilde{U}=-i \frac{\left|z^{n}+\int_{0}^{t} \partial^{3} z^{n} \mathrm{~d} \tau_{1}+\cdots\right|^{2} \bar{\alpha}-\left(z^{n}+\int_{0}^{t} \bar{\partial}^{3} \bar{z}^{n} \mathrm{~d} \tau_{1}+\cdots\right) \bar{\beta}}{|\alpha|^{2}+|\beta|^{2}}-i \frac{\left(z^{n}+\int_{0}^{t} \partial^{3} z^{n} \mathrm{~d} \tau_{1}+\cdots\right) \beta+\alpha}{|\alpha|^{2}+|\beta|^{2}}
$$

The polynomial in (14) obtained by Theorem 1 (proved in the next section) satisfies problem (1)-(2), and solution (15) satisfies the $\mathrm{mVN}$ equation by the Moutard transformations (see Theorem 3).

\section{Main Results}

In this section, we will prove Theorems 1 and 2

$$
\begin{aligned}
& \text { Proof of Theorem } \\
& \psi_{0}, \ldots, p_{n}=A^{n} \psi_{0} \text {. } \\
& \begin{aligned}
\widetilde{\psi} & =\psi_{0}+\int_{0}^{t} A p_{0} \mathrm{~d} \tau_{0}+\int_{0}^{t} A p_{1} \mathrm{~d} \tau_{1}+\cdots \\
& =\psi_{0}+t A \psi_{0}+\frac{t^{2}}{2} A^{2} \psi_{0}+\cdots
\end{aligned}
\end{aligned}
$$

Hence by using Taylor series of $e^{t A}$, we obtain

$$
\widetilde{\psi}=e^{t A} \psi_{0},
$$

which is the formal solution of the problem (1)-(2).

For example, initial data (2) could be given by one of the following minimal surfaces [11].

(1) Higher order Enneper surface:

$$
\begin{aligned}
\left(\psi_{1}, \psi_{2}\right)= & \left(z^{n}, 1\right) \\
\widetilde{\psi}= & z^{n}+n(n-1)(n-2) z^{n-3} t \\
& +n(n-1) \cdots(n-5) z^{n-6} \frac{t^{2}}{2 !}++n(n-1) \cdots \\
& (n-8) z^{n-9} \frac{t^{3}}{3 !}+\cdots
\end{aligned}
$$

(2) Catenoid:

$$
\begin{aligned}
\psi_{1} & =\frac{e^{-(z / 2)}}{\sqrt{2}}, \\
\psi_{2} & =\frac{e^{\bar{z} / 2}}{\sqrt{2}}, \\
\widetilde{\psi}: \psi_{1}(z, \bar{z}, t) & =\frac{e^{-(z / 2)-(t / 8)}}{\sqrt{2}}, \\
\psi_{2}(z, \bar{z}, t) & =\frac{e^{(\bar{z} / 2)-(t / 8)}}{\sqrt{2}} .
\end{aligned}
$$

(3) Helicoid:

$$
\begin{aligned}
\psi_{1} & =\frac{e^{-(z / 2)}}{\sqrt{2}}, \\
\psi_{2} & =\frac{i e^{\bar{z} / 2}}{\sqrt{2}}, \\
\widetilde{\psi}: \psi_{1}(z, \bar{z}, t) & =\frac{e^{-(z / 2)-(t / 8)}}{\sqrt{2}}, \\
\psi_{2}(z, \bar{z}, t) & =i \frac{e^{(z / 2)+(t / 8)}}{\sqrt{2}},
\end{aligned}
$$

which satisfy (1).

Proof of Theorem 2. Let us prove that $|\alpha|^{2}+|\beta|^{2}=a^{2}+b^{2}+c^{2} \Longleftrightarrow x=y=0$, for $n \geq 3$. 


$$
\begin{aligned}
|\beta|^{2}= & \beta \bar{\beta}=\int_{0}^{z} \psi_{1}^{2} \mathrm{~d} z \int_{0}^{\bar{z}} \bar{\psi}_{1}^{2} \mathrm{~d} \bar{z}+\int_{0}^{t} k \mathrm{~d} \tau \int_{0}^{t} \bar{k} \mathrm{~d} \tau \\
& -\int_{0}^{z} \psi_{1}^{2} \mathrm{~d} z \int_{0}^{t} \bar{k} \mathrm{~d} \tau-\int_{0}^{t} k \mathrm{~d} \tau \int_{0}^{t} \bar{\psi}_{1}^{2} \mathrm{~d} \bar{z} \\
& -2 i(a-b i)\left(\int_{0}^{z} \psi_{1}^{2} \mathrm{~d} z-\int_{0}^{t} k \mathrm{~d} \tau\right) \\
& -2 i(a-b i)\left(\int_{0}^{t} \bar{k} \mathrm{~d} \tau-\int_{0}^{\bar{z}} \bar{\psi}_{1}^{2} \mathrm{~d} \bar{z}\right)+4\left(a^{2}+b^{2}\right),
\end{aligned}
$$

where $\psi_{1}$ is given by (14) and $k$ has the following form:

$$
\begin{aligned}
k= & \left(n z^{n-1}+n(n-1)(n-2)(n-3) z^{n-4} t \ldots\right)^{2} \\
& -2 \psi_{1}\left(n(n-1) z^{n-2}+n(n-1)(n-2)(n-3)(n-4) z^{n-5} t\right) .
\end{aligned}
$$

For $x=y=0,|\beta|^{2}=4\left(a^{2}+b^{2}\right)$ and $|\alpha|^{2}=4 c^{2}$.

If you give large parameters $a \gg 1, b \gg 1$, and $c \gg 1$ for moving surfaces of Enneper of higher order $\Sigma \longrightarrow \Sigma_{a, b, c}$ by a predetermined straight line, then the soliton deformation [2] of the surfaces $S_{a, b, c}$ is defined globally; that is, all surfaces are deformed into the saddle surface. Potentials $\widetilde{U}$ in this case are regular by Theorem 4.2. Also, the regularity of the surface follows the inverse minimal surfaces that are regular at the point $x=0, y=0$ :

$$
\begin{aligned}
& \tilde{U}_{x} \neq 0, \\
& \tilde{U}_{y} \neq 0, \\
& \tilde{U}_{t} \neq 0 .
\end{aligned}
$$

The Gaussian curvature (with the exception of isolated points $x=y=0$ ) of surfaces takes negative values, since the soliton deformation translates all surfaces into a hyperbolic surface (more precisely to the saddle surface) (see Figures 1 and 2).

Corollary 1. For all surfaces $\sum$ with order $n=4,5,7, \ldots$ (means except the three times order), potentials $\widetilde{U}$ are regular, even though partial derivatives of potentials $\widetilde{U}$ with respect to $x$, $y$ until the $(n-1)$-th order in the isolated point $x=y=0$ of surfaces $\Sigma, n \in N, a=b=c=0$, have singular values and hold the following finite limits:

$$
\begin{aligned}
\lim _{r \longrightarrow 0, \varphi=\text { const }}\left(\frac{\partial^{2 j-1}}{\partial x^{2 j-1}}+\frac{\partial^{2 j-1}}{\partial y^{2 j-1}}\right) \tilde{U}(x, y, 0)= & \sum_{s=1}^{n_{1}}\left(a_{s} \cos 2 s \varphi\right. \\
& \left.+b_{s} \sin 2 s \varphi\right)
\end{aligned}
$$

$$
\lim _{r \longrightarrow 0, \varphi=\text { const }}\left(\frac{\partial^{2 j}}{\partial x^{2 j}}+\frac{\partial^{2 j}}{\partial y^{2 j}}\right) \tilde{U}(x, y, 0)=\sum_{s=1}^{n_{2}} c_{s} \cos 2 s \varphi,
$$

where $n_{1}=2 j$ and $n_{2}=2 j+1, j=0,1, \ldots$, respectively, $a_{s}$ and $b_{s}$ are nonzero, and $c_{s}$ is some constant $(s \in N)$.

Proof. From the potential formula of (3.6), we take partial derivatives with respect to two variables, briefly change to polar coordinates, and calculate limits at $r \longrightarrow 0, \varphi=$ const, in the corresponding orders:

$$
j=1: n_{1}=2, \lim _{r \longrightarrow 0, \varphi=\text { const }} \frac{\partial}{\partial x} \tilde{U}(x, y, 0)=-\frac{8}{3} \cos 2 \varphi+\frac{4}{3} \cos 4 \varphi,
$$

$$
\begin{aligned}
\lim _{r \longrightarrow 0, \varphi=\text { const }} \frac{\partial}{\partial y} \tilde{U}(x, y, 0) & =\frac{8}{3} \sin 2 \varphi+\frac{4}{3} \sin 4 \varphi, \\
n_{2} & =3, \lim _{r \longrightarrow 0, \varphi=\text { const }} \frac{\partial^{2}}{\partial x^{2}} \tilde{U}(x, y, 0)=-9 \cos 2 \varphi+9 \cos 4 \varphi-3 \cos 6 \varphi,
\end{aligned}
$$

$\lim _{r \longrightarrow 0, \varphi=\text { const }} \frac{\partial^{2}}{\partial y^{2}} \tilde{U}(x, y, 0)=9 \cos 2 \varphi+9 \cos 4 \varphi+3 \cos 6 \varphi$,

$$
\begin{aligned}
j & =2: n_{1}=4, \lim _{r \longrightarrow 0, \varphi=\text { const }} \frac{\partial^{3}}{\partial x^{3}} \tilde{U}(x, y, 0)=-\frac{192}{5} \cos 2 \varphi+\frac{288}{5} \cos 4 \varphi--\frac{192}{5} \cos 6 \varphi+\frac{48}{5} \cos 8 \varphi, \\
\lim _{r \longrightarrow 0, \varphi=\text { const }} \frac{\partial^{3}}{\partial y^{3}} \tilde{U}(x, y, 0) & =-\frac{192}{5} \sin 2 \varphi-\frac{288}{5} \sin 4 \varphi-\frac{192}{5} \sin 6 \varphi--\frac{48}{5} \sin 8 \varphi, \\
n_{2} & =5, \lim _{r \longrightarrow 0, \varphi=\text { const }} \frac{\partial^{4}}{\partial x^{4}} \tilde{U}(x, y, 0)=-200 \cos 2 \varphi+400 \cos 4 \varphi--400 \cos 6 \varphi+200 \cos 8 \varphi-40 \cos 10 \varphi,
\end{aligned}
$$




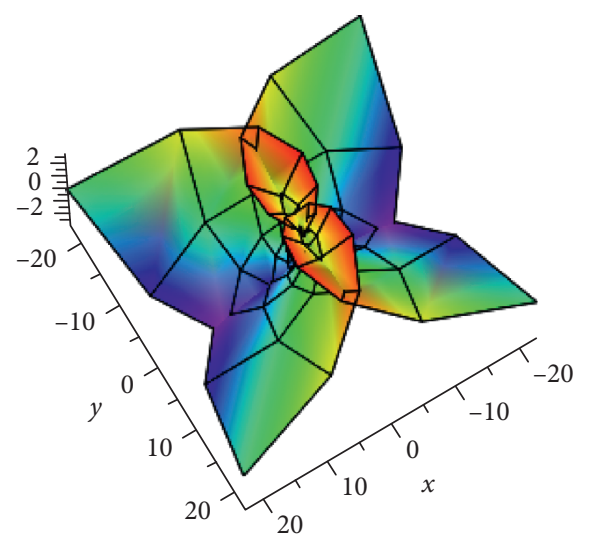

(a)

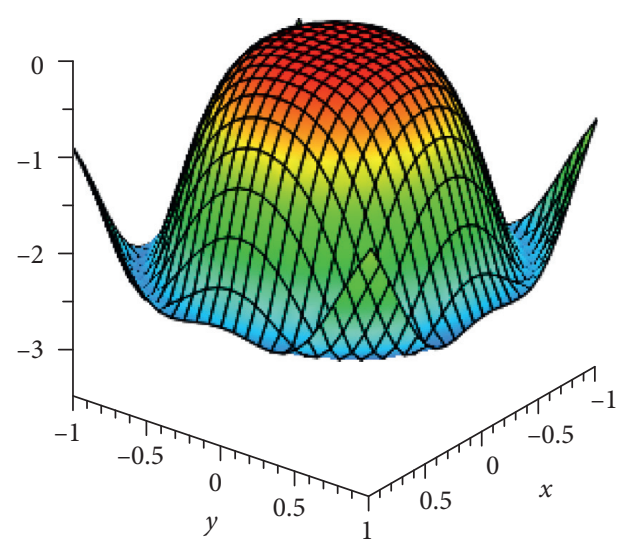

(b)

Figure 1: (a) Enneper fifth-order surface in the interval $x \in[-1.2,1.2], y \in[-1.2,1.2]$; (b) soliton deformation of inverted Enneper fifthorder surface at the point of origin for nonzero parameters $a, b, c$.

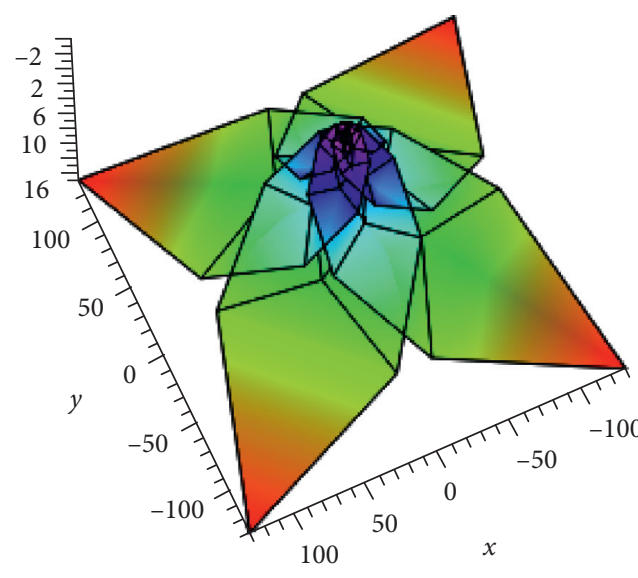

(a)

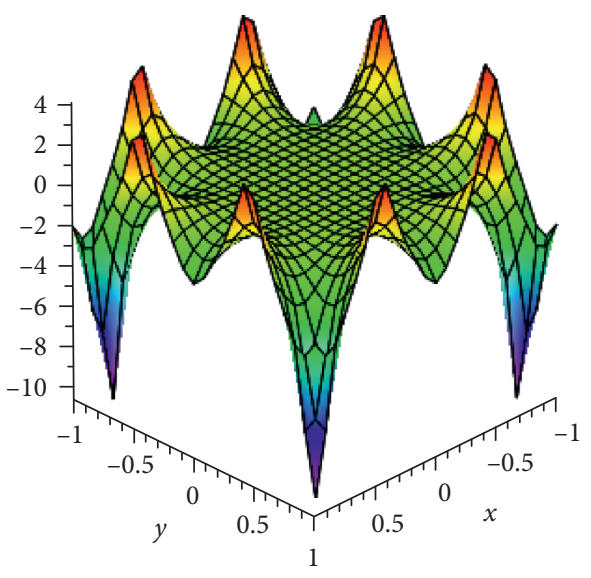

(b)

FiguRE 2: (a) Enneper seventh-order surface in the interval $x \in[-1.2,1.2], y \in[-1.2,1.2]$; (b) soliton deformation of inverted Enneper seventh-order surface at the point of origin for parameters $a=b=c=0$.

$$
\begin{aligned}
& \lim _{r \longrightarrow 0, \varphi=\text { const }} \frac{\partial^{4}}{\partial y^{4}} \tilde{U}(x, y, 0)=-200 \cos 2 \varphi-400 \cos 4 \varphi-400 \cos 6 \varphi--200 \cos 8 \varphi-40 \cos 10 \varphi ; j=3: n_{1}=6, \\
& \lim _{r \longrightarrow 0, \varphi=\text { const }} \frac{\partial^{5}}{\partial x^{5}} \tilde{U}(x, y, 0)=-\frac{8640}{7} \cos 2 \varphi+\frac{21600}{7} \cos 4 \varphi--\frac{28800}{7} \cos 6 \varphi+\frac{21600}{7} \cos 8 \varphi-\frac{8640}{7} \cos 10 \varphi+\frac{1440}{7} \cos 12 \varphi, \\
& \lim _{r \longrightarrow 0, \varphi=\text { const }} \frac{\partial^{5}}{\partial y^{5}} \tilde{U}(x, y, 0)=\frac{8640}{7} \sin 2 \varphi+\frac{21600}{7} \sin 4 \varphi+\frac{28800}{7} \sin 6 \varphi++\frac{21600}{7} \sin 8 \varphi+\frac{8640}{7} \sin 10 \varphi+\frac{1440}{7} \sin 12 \varphi,
\end{aligned}
$$

and so on.

For even and odd orders of Enneper surfaces of higher order, we obtain the identities (24) and (25).

\section{Applications in the Game Theory}

The given Kazakh proverb with the number component is "You should tumble six year camel rather than tumbling your sixty year grandfather."
In Figure 3, given T-tumble, not T-does not tumble, outCamel came out of the shed, in-Camel in the shed.

Payoffs at terminal nodes are shown in the following order:

(a) Find the backward-induction solutions

(b) Write the strategic form associated with this game

(c) What do you get when you apply the iterative deletion of weakly dominated strategies (IDWDS)? 




Figure 3: Perfect-information game.

(d) What are the Nash equilibria?

Applying the backward algorithm and IDWDS procedure [12] to a given tree, we have the following solution.

(lost, not T, T): backward solution means all players have optimal payoff after comparing payoffs in terminal nodes if Camel chooses action lost with payoff -1 . Player 1 chooses action not T with payoff 1 , and Player 2 chooses action T with payoff 100 .

The strategic form associated with this game has the following three cases:

(1) If Player 1 chooses notT and Player 2 chooses T, then Camel has win, lost actions: $(-1,1,100),(98,1,1)$

(2) If Player 1 chooses notT and Player 2 chooses notT, then Camel has out, in actions: $(100,0,0),(0,50,50)$

(3) If Player 1 chooses T, Player 2 has notT, T actions: $(99,0,1),(102,-1,-1)$

The meaning of the given proverb about the effective way for Player 2 (boy) is the Nash equilibria (lost, notT, T) with payoffs $(-1,1,100)$ where number -1 is the most optimal payoff for Camel. But this strategy is unstable for the boy; if Player 1 (grandfather) is angry and bad, then he could tumble the boy.

Here, $\mathrm{mVN}$ equations are applied as the solution method as follows:

Moutard or Darboux transformations [6] work for initial given solutions; furthermore, other solutions could be found. This result is significant for the Kazakh proverb with the number component as backward solutions; Nash equilibria were found in terms of trees as Figure 3. Likewise, other hundreds of examples with Kazakh proverbs could be represented as a rooted tree in game theory.

This example illustrates connection between mathematics and Kazakh proverbs. In addition, Kazakh proverbs have undoubted relation to the Muslim religion [13]. However, because of a huge number of historical facts [14], the readers can find alternative properties of mathematics and religion. The author means any other examples (given below) will adhere to the following paradoxical law:

$$
A_{1} \bar{R} A_{2} \text { and } A_{2} R A_{3} \longrightarrow A_{1} R A_{3} \text {, }
$$

where in $A_{1} \bar{R} A_{2}, A_{1}$ and $A_{2}$ do not have the relation.

This law (27) illustrates the main result of this work as follows.

Let $A_{1}$ be solutions of $\mathrm{mVN}$ equations, $A_{2}$ be minimal surfaces with singular points, and $A_{3}$ be minimal surfaces with regular points. This assignment satisfies the law (27) for every order of surfaces $n \geq 3$ because there is no relation between all solution and minimal surfaces with singular points and $A_{2} R A_{3}$ shows relation between minimal surfaces with singular and regular points (see Corollary 1), implying $A_{1} R A_{3}$ as solutions of $\mathrm{mVN}$ equations related to minimal surfaces with regular points (see Theorem 2).

For $n=1,2$, the nonassociative law: $A_{1} R A_{2}, A_{2} \bar{R} A_{3} \longrightarrow A_{1} \bar{R} A_{3}$, is satisfied.

The purpose of $\mathrm{mVN}$ equation application as the solution in the game theory is as follows.

Blowing-up or singular solutions of $\mathrm{mVN}$ equations led to regular solutions of $\mathrm{mVN}$ equations for every order of surfaces $n \geq 3$, as initial nonlinear $\mathrm{mVN}$ equations led to the linear system of equations, and finally, the alternative between mathematics and religion led to the relation between mathematics and Kazakh proverbs.

In the future, this procedure will show a solution to some nonlinear disagreements or any other life circumstances.

In summary, scientists could find some set of relations in which all components satisfy the paradoxical law (27). Given about five components, e.g., $A_{1}, A_{2}, A_{3}, A_{4}$, and $A_{5}$, the readers may concentrate on one component and try to solve 
this problem (about 20 percent of all components) to improve the result of some goal and may gain about 80 percent efficiency according to the Pareto efficiency law.

As this law contradicts the Pareto efficiency law, it is named "paradoxical."

\section{Conclusions}

In conclusion, to solve the mVN equation, Theorem 1 was used not only with Enneper surfaces but also with other minimal surfaces as an example. The obtained results in this article (Theorem 2 and Corollary 1) were graphically represented and introduced to a paradoxical law of game theory. Further analysis of the potentials led to the study of Kazakh proverbs, which are rich in educational and meaningful words, presented in the form of a tree in game theory. Although the meaning of these two concepts as proverbs and potentials (not in an analytical form) is different, the readers can be sure about the fulfillment of paradoxical law in studies of the structures of these objects. The meaning of this law is that nonlinear objects can be reduced to the linear form, and blowing-up solutions can be reduced to the regular form in general. Therefore, the Darboux transformation and potentials are also applied in game theory.

\section{Data Availability}

The author confirms that the data supporting the findings of this study are available in this published article.

\section{Conflicts of Interest}

The author declares that there are no conflicts of interest.

\section{Acknowledgments}

This work was partially supported by Suleyman Demirel University.

\section{References}

[1] A. Doliwa and A. M. Grundland, "Minimal surfaces in the soliton surface approach," 2015, https://arxiv.org/abs/1511. 02173.

[2] V. B. Matveev and M. A. Salle, Darboux Transformation and Solitons, Springer-Verlag, Berlin, Germany, 1991.

[3] W.-Q. Peng, S.-F. Tian, and T.-T. Zhang, "Dynamics of breather waves and higher-order rogue waves in a coupled nonlinear Schrödinger equation," EPL (Europhysics Letters), vol. 123 , no. 5 , p. $50005,2018$.

[4] L. Ling, L.-C. Zhao, and B. Guo, "Darboux transformation and classification of solution for mixed coupled nonlinear Schrödinger equations," Communications in Nonlinear Science and Numerical Simulation, vol. 32, pp. 285-304, 2016.

[5] L. V. Bogdanov, "Veselov-Novikov equation as a natural twodimensional generalization of the Korteweg-de Vries equation," Theoretical and Mathematical Physics, vol. 70, no. 2, pp. 219-223, 1987.

[6] D. Yu, Q. P. Liu, and S. Wang, "Darboux transformation for the modified Veselov-Novikov equation," Journal of Physics A: Mathematical and General, vol. 35, no. 16, pp. 3779-3785, 2001.
[7] I. A. Taimanov, "Blowing up solutions of the modified Novikov-Veselov equation and minimal surfaces," Theoretical and Mathematical Physics, vol. 182, no. 2, pp. 173-181, 2015.

[8] I. A. Taimanov, "The moutardtransformation of two-dimensional Dirac operators and MoËbiusgeometry," Mathematical Notes, vol. 97, no. 1-2, pp. 124-135, 2015.

[9] S.V. Manakov, "The method of the inverse scattering problem and two dimensional evolution equations," UspekhiMatematicheskikhNauk, vol. 31, pp. 245-246, 1976.

[10] I. A. Taimanov, "Two-dimensional Dirac operator and the theory of surfaces," Russian Mathematical Surveys, vol. 61, no. 1, pp. 79-159, 2006.

[11] J. Oprea, Differential Geometry and Its Applications, Mathematical Association of America, Washington, DC, USA, 2nd edition, 2007.

[12] G. Bonanno, Game Theory, University of California, Davis, CA, USA, 2018.

[13] C. M. Y. Malgajuly, Religion and Tradition, Almaty, Kazakhstan, 2013.

[14] A. Nysanbaev, Mathematics and Religion, Alma-Ata, Kazakhstan, 1966. 